Title: Astrocytes are implicated in BDNF-mediated enhancement of hippocampal longterm potentiation

Authors: Joana I. Gomes ${ }^{1,2, \#, ~ J o a ̃ o ~ J e s u s ~}{ }^{1,2, \#, ~ R e n a t a ~ M a c a u ~}{ }^{1,2}$, Joana GonçalvesRibeiro ${ }^{1,2}$, Sara Pinto ${ }^{1,2}$, Carolina Campos Pina ${ }^{1,2}$, Adam Armada-Moreira ${ }^{1,2,3}$, Ana Maria Sebastião ${ }^{1,2}$, Sandra H. Vaz ${ }^{1,2, *}$

\title{
Affiliations:
}

1 Instituto de Medicina Molecular João Lobo Antunes, Faculdade de Medicina da Universidade de Lisboa, Av. Prof. Egas Moniz, 1649-028 Lisbon, Portugal.

${ }^{2}$ Instituto de Farmacologia e Neurociências, Faculdade de Medicina da Universidade de Lisboa, Av. Prof. Egas Moniz, 1649-028 Lisbon, Portugal.

${ }^{3}$ Interdisciplinary Nanoscience Center (iNANO), Aarhus University, Gustav Wieds Vej 14, 8000 Aarhus, Denmark.

*Correspondence: Sandra H. Vaz, Instituto de Medicina Molecular João Lobo Antunes, Faculdade de Medicina da Universidade de Lisboa, Av. Prof. Egas Moniz, 1649-028 Lisbon, Portugal.svaz@medicina.ulisboa.pt

\section{\#Co-first authors}

\section{Abstract}

It is known that astrocytes, by the $\mathrm{Ca}^{2+}$-dependent release of gliotransmitters, which then act in pre- and post-synaptic receptors, modulate neuronal transmission and plasticity. Thus, hippocampal $\theta$-burst long-term potentiation (LTP), which is a form of synaptic plasticity, can be modulated by astrocytes, since these cells release gliotransmitters that are crucial for the maintenance of LTP. Therefore, in this study, we hypothesized that the facilitatory action of BDNF upon LTP would involve astrocytes. To address that possibility, fEPSP recordings were performed in CA3-CA1 area of hippocampal slices from three different experimental models: Wistar rats where astrocytic metabolism was selectively reduced by a gliotoxin, the DL-fluoricitric acid (FC), IP3R2 ${ }^{-/}$mice, which lack IP3R2-mediated $\mathrm{Ca}^{2+}$-signaling in astrocytes and dn-SNARE transgenic mice, in which the SNARE-dependent release of gliotransmittersis impaired. For the three models we observed that the astrocytic impairment abolished the excitatory BDNF effect upon hippocampal LTP, only while inducing LTP with a mild $\theta$-burst stimulation paradigm. The present data shows for the first time that astrocytes play an active role in the facilitatory action of BDNF upon LTP, depending on stimulation paradigm. 
Keywords: Astrocyte-neuron communication; gliotransmission; BDNF; long-term potenciation.

\section{Introduction}

Astrocytes, the main glial cell type in central nervous system (CNS), are in close contact with neurons leading to the recognition of a bidirectional communication between both neurons and astrocytes at the synapse (Araque et al., 1999). These cells play a crucial modulatory role upon synaptic function since they respond to neurotransmitters through intracellular $\mathrm{Ca} 2+$ transients, that induce the release of gliotransmiters, which modulate neuronal excitability and synaptic function (Kofuji et al., 2021). Among other functions, astrocytes modulate long-term potentiation (LTP), the neurophysiological basis for learning and memory (Bliss et al., 1993), through the release of ATP (Pascual et al., 2005), glutamate (Perea and Araque, 2007) and D-serine (Henneberger et al., 2010). CA1 hippocampal LTP can be triggered when postsynaptic activity and astrocytic $\mathrm{Ca}^{2+}$ dependent glutamate release occur at the same time (Perea et al., 2007). The $\mathrm{Ca}^{2+}$ dependent release of D-serine from CA1 hippocampal astrocytes controls NMDARdependent plasticity in the excitatory synapses nearby (Henneberger et al., 2010). The extracellular adenosine, derived from astrocytic ATP (Bal-Price et al., 2002; Lalo et al., 2014), regulates synaptic transmission and modulates LTP (Pascual et al., 2005).

Brain-derived neurotrophic factor (BDNF) is a neurotrophin with essential functions in neuronal survival, differentiation, and synaptic plasticity(G. Leal et al., 2017; Sebastião et al., 2011) that mediates its effects through the activation of TrkB receptors. Different TrkB receptor isoforms are generated by alternative splicing, namely one full-length form of TrkB (TrkB-fl) and two truncated TrkB-t (e.g. Sebastião et al., 2011) isoforms. The enhancement of LTP by BDNF (Korte et al., 1995; Figurov et al., 1996; Patterson et al., 1996; Fontinha et al., 2008) is achieved through the activation of TrkB-fl receptors (Xu et al., 2000; Minichiello et al., 2002), since hippocampal LTP is strongly impaired in both BDNF (Gottschalk et al., 1999; Korte et al., 1995; Patterson et al., 1996) and TrkB (Xu et al., 2000; Minichiello et al., 2002) knockout mice. Besides BDNF effects on LTP, it has also been shown that in cultured astrocytes, BDNF mediates rapid $\mathrm{Ca}^{2+}$ transient through TrkB-t receptor, that results from G protein-dependent PLC activation and release of $\mathrm{Ca}^{2+}$ from Ins(1,4,5)P3-sensitive stores (Rose et al., 2003). In neuromuscular junction was shown that BDNF elicits a $\mathrm{Ca}^{2+}$ response in perisynaptic Schwann cells 
thought TrkB-t receptor (Todd et al., 2007). Moreover, BDNF overexpression from astrocytes lead to an improvement of cognitive performance in an Alzheimer's mice model (De Pins et al., 2019). Besides astrocytic BDNF-mediated modulation of $\mathrm{Ca}^{2+}$ transients, this neurotrophic factor is able to induce the release of glutamate from astrocytes through a $\mathrm{Ca}^{2+}$ dependent mechanism (Pascual et al, 2001), which may contribute to the enhancement of LTP.

Although it is well established that both BDNF and astrocytes modulate LTP, nothing is known about the role that astrocytes may play upon BDNF regulation of LTP. Here, we have investigated the functional role of astrocytes upon the enhancement of hippocampal LTP by BDNF in three complementary approaches: i) under the pharmacological astrocytic blockade with the gliotoxin fluorocitrate (FC) (Swanson et al., 1994); ii) in a transgenic mice that lacks astrocytic release of gliotransmitters (dn-SNARE mice) (Pascual et al., 2005); and iii) in a transgenic mice that lacks astrocytic $\mathrm{Ca}^{2+}$ signalling (IP3R2 ${ }^{-/-}$mice) (Li et al., 2005). By performing electrophysiological recordings, in these three considered approaches, we unveiled that astrocytes control the effect of BDNF upon synaptic plasticity in the hippocampus. We thus identified a new role of astrocytes in the CNS - to fine-tune synaptic plasticity mediated by neurotrophins.

\section{Methods}

\section{Experimental models}

Animals were maintained in controlled temperature $\left(21 \pm 1^{\circ} \mathrm{C}\right)$ and humidity $(55 \pm 10 \%)$ conditions with a 12:12 h light/dark cycle and access to food and water ad libitum. All procedures were carried out according to the European Union Guidelines for Animal Care (European Union Council Directive 2010/63/EU) and Portuguese law (DL 113/2013) with the approval of the Institutional Animal Care and Use Committee. Care was taken to minimize the number of animals sacrificed.

Generation of 1,4,5-triphosphate (IP3) type-2 receptor (R2) knockout (IP3R2--) mice. A mouse strain with targeted deletion of Itpr2 gene was kindly supplied for this project by Prof. João F. Oliveira (U. Minho, Portugal) (Guerra-Gomes et al., 2020), under agreement with Prof. Ju Chen (U.C. San Diego, USA) (Li et al., 2005), who have generate the IP3R2 ${ }^{-/}$mice. IP3R2 ${ }^{-/}$and their respective littermate WT controls (IP3R ${ }^{+/+}$) were obtained by mating couples of IP3R2 ${ }^{+/}$. Collected toe tissue was used for DNA extraction and subsequent genotyping by polymerase chain reaction (PCR) analysis using WT (Forward (F), 5'- ACCCTGATGAGGGAAGGTCT-3'; Reverse (R), 5'ATCGATTCATAGGGCACACC-3') and mutant allele specific primers (neo-specific 
primer:

$\mathrm{F}$,

5'-AATGGGCTGACCGCTTCCTCGT-3';

$\mathrm{R}$

5'-

TCTGAGAGTGCCTGGCTTTT-3') as previously described(Li et al., 2005).

Generation of dn-SNARE mice. A dn-SNARE strain was kindly supplied for this project by Prof. João F. Oliveira (U. Minho, USA) (Sardinha VM et al., 2017), under agreement with Prof. Philip Haydon (Tufts U., USA) (Pascual et al., 2005), and were maintained in the C57BI6/J genetic background. The generation of dn-SNARE mice was performed as previously described (Pascual et al., 2005). Briefly, the dn-SNARE mice and wild-type (WT) littermates were obtained by crossing two distinct transgenic mouse lines: hGFAP.tTA mice, where the expression of tetracycline transactivator (tTA) is mediated by the astrocyte-specific human glial fibrillary acidic protein (hGFAP) promoter and tetO.dnSNARE, in which the dominant-negative domain of vesicular SNARE VAMP2/synaptobrevin II, as well as, the reporter gene for enhanced green fluorescence protein (EGFP) are co-expressed under the control of the tetracycline operator (tetO). The generated animals will express Lac-Z, EGFP and SNARE transgenes on their astrocytes, but not on their neurons which leads to the selective impairment of vesicular gliotransmitters release from astrocytes. The conditional expression of the dnSNARE transgenes caused interference with the SNARE complex formation and consecutive blockade of exocytosis specifically in astrocytes (astrocytes derived from dnSNARE mice displayed a $91 \%$ reduction in the number of fusion events) (Sultan et al., 2015), impairing the vesicular release of gliotransmitters. Doxycycline (Dox, Sigma-Aldrich, St. Louis, Missouri, EUA) administration in the drinking water $(25 \mu \mathrm{L} / \mathrm{mL})$, prevents the transgene expression, consequently leading to the inhibition of SNARE, Lac-Z and EGFP expression, resulting in the functional gliotransmission process. Animals genotype was confirmed by PCR analysis, where mice negative (wild-type) or positive for both transgenes (dn-SNARE) were tested, while mice expressing only single transgenes (GFAP-tTA or tetO.dnSNARE) were not included. Collected toe tissue was used for DNA extraction and subsequent genotyping by PCR analysis using primers to identify the tTA, tetO and HSF-1 genes: tTA-F, 5'-ACTCAGCGCTGTGGGGCATT-3' and tTA-R, 5'GGCTGTACGCGGACCCACTT-3'; tetO-F, 5'-TGGATAAAGAAGCTCATTAATTGTCA3' and tetO-R, 5'-GCGGATCCAGACATGATAAGA-3'; HSF-1-F, 5'TCTCCTGTCCTGTGTGCCTAGC-3' and HSF-1-R, 5' CAGGTCAACTGCCTACACAGACC.

\section{Hippocampal slices preparation}


Hippocampal slices were prepared as routinely in our lab (Rei et al., 2020) from Wistar rats, IP3R2-KO and dn-SNARE mice with 8-12 weeks old. Wistar rats (Charles River, Barcelona, Spain) were deeply anesthetized with isoflurane (Esteve, Barcelona, Spain) and sacrificed by decapitation for hippocampal slice preparation. IP3R2-KO and dnSNARE mice were sacrificed by decapitation after cervical displacement and the brain was rapidly removed in order to prepare hippocampal slices. The hippocampi were dissected in ice-cold artificial cerebrospinal fluid (aCSF) containing (in mM): $124 \mathrm{NaCl}, 3$ $\mathrm{KCl}, 1.2 \mathrm{NaH}_{2} \mathrm{PO}_{4}, 25 \mathrm{NaHCO}_{3}, 2 \mathrm{CaCl}_{2}, 1 \mathrm{MgSO} 4$ and 10 glucose), which was continuously gassed with $95 \% \mathrm{O}_{2}$ and $5 \% \mathrm{CO}_{2}$. The hippocampal slices were quickly cut perpendicularly to the long axis of the hippocampus ( $400 \mu \mathrm{m}$ thick) with a Mcllwain tissue chopper and allowed to recover functionally and energetically for at least $1 \mathrm{~h}$ in a resting chamber filled with continuously oxygenated aCSF, at room temperature $\left(22-25^{\circ} \mathrm{C}\right)$, before being set up for electrophysiological recordings.

\section{Extracellular recordings of fEPSPs}

Following the recovery period, slices were transferred to a recording chamber for submerged slices ( $1 \mathrm{ml}$ capacity plus $5 \mathrm{ml}$ dead volume) and were constantly superfused at a flow rate of $3 \mathrm{ml} / \mathrm{mim}$ with aCSF kept at $32^{\circ} \mathrm{C}$, gased with $95 \% \mathrm{O}_{2} / 5 \% \mathrm{CO}_{2}$. Evoked field excitatory postsynaptic potentials (fEPSP) were recorded extracellularly by using a microelectrode (4 to $8 \mathrm{M} \Omega$ resistance) filled with aCSF solution placed in the stratum radiatum of the CA1 area. fEPSP data were acquired using an Axoclamp-2B amplifier (Axon Instrumnets, Foster City, CA). fEPSPs were evoked by stimulation through a concentric electrode to the Schaffer collateral fibres. Each individual stimulus consisted of a $0.1 \mathrm{~ms}$ rectangular pulse applied once every 20s, except otherwise indicated. Averages of six consecutive responses were continuously acquired, digitized with the WinLTP program (Anderson and Colloingridge 2001) and quantified as the slope of the initial phase of the averaged fEPSPs (and the amplitude of presynaptic fibre voley while performing input/output analysis). The stimulus intensity was adjusted at the beginning of the experiment to obtain a fEPSP slope that corresponds to about $50 \%$ of the maximal fEPSP slope.

Input/output curves. For input/output curves $(\mathrm{I} / \mathrm{O})$, after obtaining a stable baseline under the standard stimulation conditions, the stimulus intensity was increased by $20 \mu \mathrm{A}$ every 4 minutes $(60-320 \mu \mathrm{A})$. The I/O curves were plotted as the fEPSP slope against the stimulus intensity, as the presynaptic fibber volley (PSFV) amplitude against stimulus intensity, and as the fEPSP slope against PSFV amplitude, which provides a measure 
of synaptic efficiency. The maximal slope values were obtained by extrapolation upon nonlinear fitting of the $\mathrm{I} / \mathrm{O}$ curve, an F-test being used to determine differences between the parameters.

Long-term potentiation induction. LTP was induced by a applying $\theta$-burst stimulation, since this pattern of stimulation is considered to be closer to what occurs physiologically in the hippocampus during episodes of learning and memory in living animals (Albensi et al., 2007). Two different $\theta$-burst stimulation paradigms were used: one, named as strong $\theta$-burst LTP-inducing paradigm, consisting of 1 train of 15 bursts (200 ms interburst interval), each burst being composed by 4 pulses delivered at $100 \mathrm{~Hz}[1 \times(15 \times 4)]$; the other named week $\theta$-burst LTP-inducing paradigm, consisting of 1 train of 3 bursts (200 ms inter-burst interval), each burst being composed by 3 pulses delivered at 100 $\mathrm{Hz}[1 \times(3 \times 3)]$. The strong $\theta$-burst stimulation was chosen since is well documented the exogenous BDNF effect on LTP for this particular stimulus (Fontinha et al., 2008). The weak $\theta$-burst stimulation paradigm was the same used by Pascual and collaborators (2005) to demonstrate the LTP impairment in dn-SNARE mice.

After obtaining a stable recording of the fEPSP slope, one of $\theta$-burst of stimuli described were applied, and the stimulus paradigm was then resumed to pre-burst conditions up to the end of the recording period (60 min after burst stimulation). LTP magnitude was quantified as the \% change in the average slope of the fEPSP taken from 50-60 minutes after the induction of LTP as compared with the average slope of the fEPSP measured during the 10 minutes before the induction of LTP. Since each slice allows recordings from two independent pathways, LTP was recorded in the first pathway, after 60 min of LTP induction, BDNF $(20 \mathrm{ng} / \mathrm{mL})$ was added to the superfusion solution. After at least 30 min of BDNF perfusion, LTP was induced in the second pathway. BDNF remained in the bath until the end of the experiment. I/O data was acquired in a new slice by stimulating the second pathway. In any case, fEPSPs were recorded under basal stimulation conditions (standard stimulus intensity and frequency) and stability of fEPSP slope values were guaranteed for more than $10 \mathrm{~min}$ before changing any protocol parameter. One or two slices per animal were tested in each experimental day.

Drugs. BDNF was generously provided by Regeneron Pharmaceuticals (Tarrytown, NY), DL-fluorocitric acid (FCA) $(200 \mu M)$, 2-p-(2-Carboxyethyl) phenethylamino-5'-Nethylcarboxamidoadenosine (CGS 21680, 30 nM), 2-(2-Furanyl)-7-(2-phenylethyl)-7H- 
pyrazolo[4,3-e][1,2,4]triazolo[1,5-c]pyrimidin-5-amine $\quad(\mathrm{SCH} \quad 58261, \quad 50 \mathrm{nM})$ were purchased from Sigma (St. Louis, MO). CGS 21680 and SCH 58261 were made up into a $5 \mathrm{mM}$ stock solution in dimethylsulfoxide (DMSO). DL-flurocitrate was prepared as described previously(Paulsen et al., 1987) and aliquots were made up into a $3.23 \mathrm{mM}$ stock solution. BDNF was supplied in a $1.0 \mathrm{mg} / \mathrm{mL}$ stock solution in $150 \mathrm{mM} \mathrm{NaCl}, 10$ $\mathrm{mM}$ sodium phosphate buffer, and $0.004 \%$ Tween 20 . Aliquots of these stock solutions were kept frozen at $-20^{\circ} \mathrm{C}$ until further use.

Statistical analysis. Data are expressed as means \pm SEM from $n$ slices. All statistical analysis was performed using GraphPad (San Diego, CA, USA) Prism software. Twosample comparisons were made using $t$ tests or by one-way ANOVA with treatment as the between-subject factor, followed by Sidak's post hoc test for multiple comparisons when comparing multiple experimental groups. Values of $p<0.05$ were considered to account for statistically significant differences.

\section{Results}

\section{Astrocytes do not control BDNF effect upon LTP induced by strong- $\theta$-burst stimulation}

To evaluate the role of astrocyte signaling for the effect of BDNF on synaptic plasticity, LTP was assessed in the CA1-CA3 area of the hippocampal slices of Wistar rats, IP3R2I- mice and dn-SNARE mice, and their respective littermate WT controls. The $\theta$-burst stimulation of the Schaffer-collateral pathway led to an initial enhancement of the fEPSP slope, followed by a decrease of the slope towards a stabilization above the values recorded before $\theta$-burst stimulation (baseline).

In the Wistar rats, the strong $\theta$-burst stimulation protocol induced a LTP magnitude, quantified as the \% change in the average slope of the fEPSP before and 50-60 min after $\theta$-burst stimulation (see Methods), of $36.7 \pm 9.23 \%(n=5)$, while in the same slices but in the presence of BDNF $(20 \mathrm{ng} / \mathrm{mL})$ the same induction paradigm induced a LTP magnitude of $80.4 \pm 8.95 \%(n=5)$. Thus, as previously reported in literature (JerónimoSantos et al., 2014; Fontinha et al., 2008), BDNF increased significantly the magnitude of LTP (Figure 1A,C; one-way ANOVA following Holm-Sidak's post hoc test, ${ }^{* *} \mathrm{p}=0.003$, $F(3,17)=22,04 ; n=5)$. To selectively reduce the astrocytic metabolism we used the fluorocitrate (FC), a reversible toxin that inhibits the enzyme aconitase causing a disruption of the tricarboxylic acid cycle and a decrease of the exported glutamine (GIn) (Berg-Johnsen et al., 1993; Hassel et al., 1994), by interfering with the glutamate-Gln 
cycle (Largo et al., 1996; Paulsen et al., 1987; Swanson et al., 1994). In hippocampal slices of Wistar rats superfused with FC $(200 \mu \mathrm{M})$ the same stimulation paradigm induced a decrease of fEPSP slope by $-17.7 \pm 10.6 \%(n=5)$, corresponding to a marked and significant impairment of LTP when compared to hippocampal slices without FC superfusion (Figure 1B, C; one-way ANOVA following Holm-Sidak's post hoc test $\theta p=0.001, n=5)$. In Wistar rat hippocampal slices superfused with $F C(200 \mu M)$ and BDNF $(20 \mathrm{ng} / \mathrm{mL})$ the magnitude of LTP was $25.6 \pm 4.99 \%$, which corresponds to a significant LTP potentiation by BDNF (Figure 1B, C one-way ANOVA following HolmSidak's post hoc test ${ }^{* *} \mathrm{p}=0.003, \mathrm{n}=6$ ).

The role of astrocytes upon synaptic plasticity is dependent of intracellular $\mathrm{Ca}^{2+}$ signaling (Henneberger et al., 2010) and gliotransmitter release (Pascual et al., 2005). Since we observed that FC, due to its toxicity, completely impaired LTP, in the following experiments we used two different animal models that have impaired astrocytic $\mathrm{Ca}^{2+}$ signaling and gliotransmitter release, respectively, to further infer on the function of astrocytes for BDNF effect on synaptic plasticity. While performing fEPSPs recordings in IP3R2-/- mice ( $\mathrm{Li}$ et al., 2005), which lacks IP3-mediated $\mathrm{Ca}^{2+}$ increases in astrocytes, and the respective littermate WT controls $\left(\mathrm{IP} 3 \mathrm{R} 2^{+/+}\right)$, we observed that the strong- $\theta$-burst stimulation applied in CA3-CA1 area of hippocampal slices from IP3R2 ${ }^{+/+}$mice induced a significant LTP magnitude increase of $43.7 \pm 5.9 \%(n=8)$ while in the same slices but in the presence of BDNF $(20 \mathrm{ng} / \mathrm{mL})$ the same induction paradigm induced a LTP magnitude of $80.4 \pm 7.44 \%(n=7)$, corresponding to a significant LTP potentiation mediated by BDNF (Figure 1D, F; one-way ANOVA following Holm-Sidak's post hoc test; $\left.{ }^{*} \mathrm{p}=0.010, \mathrm{~F}(3,18)=6.914, \mathrm{n}=7-8\right)$. Strong- $\theta$-burst stimulation applied in hippocampal slices obtain from IP3R2 ${ }^{-/-}$mice, result in an increase of fEPSP slope of $36.4 \pm 5.6 \%(n=4)$ in the absence of BDNF $(20 \mathrm{ng} / \mathrm{mL})$, while in its presence of $80.8 \pm 21.3 \%(n=3)$, which corresponds to a significant increase in LTP magnitude (Figure 1E, F; one-way ANOVA following Holm-Sidak's post hoc test; ${ }^{\mathrm{p}} \mathrm{p}=0.031, \mathrm{n}=3-4$ ).

In order to study the influence of gliotransmitters release on the BDNF effect upon hippocampal LTP we have used the dn-SNARE mice model which conditionally lacks astrocytic release of gliotransmitters by exocytosis. This conditional blockade of gliotransmitters release is achieved through the conditional expression of the dominant negative domain of vesicular SNARE protein synaptobrevin II (dn-SNARE), which interferes with the SNARE complex formation, impairing vesicular release (Sardinha et al., 2017; Pascual et al., 2005), being the expression of dn-SNARE transgene abolish by administration of doxycycline (DOX) in the drinking water of the animals. A strong $\theta$-burst 
stimulation applied to hippocampal slices from WT (DOX+) mice result in an increase of fEPSP slope of $43.9 \pm 5.28 \%(n=8)$, whereas in the same slices but in the presence of BDNF $(20 \mathrm{ng} / \mathrm{mL})$ the same induction paradigm enhanced the fEPSP slope to $63.3 \pm 1.99 \%(n=5)$, corresponding to a significant potentiation of LTP magnitude (Figure $1 \mathrm{G}$, I; one-way ANOVA following Holm-Sidak's post hoc test; * $\mathrm{p}=0.049 ; \mathrm{F}(5,24)=5.79$; $\mathrm{n}=5-8)$. For dn-SNARE (DOX+) animals, which release gliotransmitters in an exocytosis dependent mechanism, similar to WT animals, the magnitude of LTP for the same LTP induction protocol was $30.5 \pm 6.87 \%(n=5)$ in the absence of BDNF $(20 \mathrm{ng} / \mathrm{mL})$, while in its presence was $5523 \pm 7.65 \%(n=3)$, that corresponds to a significant LTP potentiation (Supp. 1 A, C; unpaired t-test, $\left.{ }^{*} p=0.024, n=3-5\right)$. The obtained results in hippocampal slices from dn-SNARE (DOX-) mice, which lacks the release of gliotransmitters by exocytosis, show that the magnitude of the LTP, evoked by strong $\theta$-burst stimulation, was $26.9 \pm 4.36 \%(n=6)$ in the absence of BDNF $(20 \mathrm{ng} / \mathrm{mL})$, while in its presence was $51.5 \pm 9.87 \%(n=4)$, which corresponds to a significant LTP potentiation (Figure $1 \mathrm{H}$, I; one-way ANOVA following Holm-Sidak's post hoc test; * $\mathrm{p}=0.032, \mathrm{n}=4-6)$. Altogether the results obtain from hippocampal slices of Wistar rats in presence of FC, IP3R2-- mice and dn-SNARE mice have shown that a strong $\theta$-burst stimulation induces a significant LTP that is significantly potentiated by the presence of BDNF.

\section{Astrocytes contrinute to the BDNF effect upon LTP induced by mild- $\theta$-burst stimulation}

Since the ability of BDNF to facilitate LTP is more prominent when LTP is induced by a weaker than by a stronger $\theta$-burst paradigm, next we explored if astrocytes could control BDNF effect upon hippocampal LTP while using a mild $\theta$-burst stimulation to induce LTP. A mild- $\theta$-burst stimulation applied in CA3-CA1 area of hippocampal slices from Wistar rats enhanced the fEPSP slope by $15.5 \pm 5.9 \%(n=7$, Figure $2 A, C)$. In the same slices but in the presence of BDNF $(20 \mathrm{ng} / \mathrm{mL})$, mild- $\theta$-burst stimulation increased fEPSP slope by $40.9 \pm 5.0 \%(n=6$, Figure $2 A, C)$ which corresponds to a significant LTP potentiation mediated by BDNF as previously reported (Fontinha et al., 2008; Jerónimo-Santos et al., 2014) (Figure 2A, C; one-way ANOVA following Holm-Sidak's post hoc test; " $\mathrm{p}=0.024$; F $(5,36)=11.83 ; n=6-7)$. In hippocampal slices of Wistar rats superfused with FC (200 $\mu \mathrm{M})$ the same mild stimulation paradigm induced a decrease of fEPSP slope by $11.7 \pm 6.70 \%(n=12)$, corresponding to a marked and significant impairment of LTP when compared to hippocampal slices without FC superfusion (Figure 2A, B, C; one-way ANOVA following Holm-Sidak's post hoc test; $\theta p=0.006, n=7-12)$. In slices superfused with FC, the presence of BDNF $(20 \mathrm{ng} / \mathrm{mL})$ induced a LTP magnitude of $-15.5 \pm 6.38 \%$ $(n=7)$, corresponding to a significant loss of excitatory BDNF action on LTP in the 
presence of FC (Figure 2B, C; one-way ANOVA following Holm-Sidak's post hoc test; $\left.{ }^{* * *} \mathrm{p}<0.001, \mathrm{n}=6-7\right)$.

Moreover, in hippocampal slices from IP3R $2^{+++}$mice, mild- $\theta$-burst stimulation induced a significant LTP magnitude increase of $47.7 \pm 8.01 \%(n=7$, Figure $2 E, F)$. In the same slices, in the presence of BDNF $(20 \mathrm{ng} / \mathrm{mL})$, the same stimulation paradigm increased the fEPSP slope by $83.3 \pm 9.61 \%$ (Figure 2D, F; one-way ANOVA following Holm-Sidak's post hoc test; ${ }^{* *} \mathrm{p}=0.006, \mathrm{n}=7$ ). Mild- $\theta$-burst stimulation applied in hippocampal slices from IP3R $2^{-/-}$in the absence of result in an increase of fEPSP of $39.2 \pm 2.35 \%$, while in BDNF (20ng/mL) presence LTP magnitude was $29.0 \pm 7.07 \%$ (Figure 2E, F; one-way ANOVA following Holm-Sidak's post hoc test; $n . s, p=0.548, n=7$ ), which corresponds to an impairment of excitatory BDNF effect upon LTP magnitude, indicating that IP3R2mediated $\mathrm{Ca}^{2+}$-signaling controls BDNF effect on hippocampal LTP while using mild- $\theta$ burst stimulation paradigm.

While recording fEPSPs from hippocampal slice of WT $(\mathrm{DOX}+)$ mice, a Mild- $\theta-b u r s t$ stimulation potentiated the fEPSP slope by $26.4 \pm 13.1 \%(n=4)$, whereas in the same slices but in the presence of BDNF $(20 \mathrm{ng} / \mathrm{mL})$ the same induction paradigm enhanced the fEPSP slope by $57.4 \pm 6.74 \%$ (Figure $2 \mathrm{G}$, I; one-way ANOVA following Holm-Sidak's post hoc test; ${ }^{*} \mathrm{p}=0.031, \mathrm{n}=4$ ) corresponding to a significant effect of BDNF on synaptic plasticity. For dn-SNARE (DOX+) mice we observed a LTP enhancement similar to the WT $(D O X+)$ mice, being the LTP magnitude of $23.2 \pm 13.9 \%(n=4)$ in the absence of BDNF $(20 \mathrm{ng} / \mathrm{mL})$ and by $50.4 \pm 8.89 \%(\mathrm{n}=4)$ in its presence (Supp. Fig. $1 \mathrm{D}, \mathrm{F}$; unpaired t-test ${ }^{*} \mathrm{p}=0.04, \mathrm{n}=4$ ). In slices from dn-SNARE (DOX-) mice, the mild- $\theta$-burst stimulation increase LTP magnitude by $23.8 \pm 4.19 \%(n=4)$ in the absence of BDNF $(20 \mathrm{ng} / \mathrm{mL})$ and by $28.9 \pm 2.7 \%(n=3)$ in its presence (Figure $2 \mathrm{H}$, I; one-way ANOVA following HolmSidak's post hoc test; n.s, $\mathrm{p}=0.724, \mathrm{n}=3-4$ ), which corresponds to a loss of BDNF action in LTP.

These results show that the compromised astrocytic function, achieved by decreasing astrocytic metabolism, compromising intracellular $\mathrm{Ca}^{2+}$-signaling or compromising the release of gliotransmitters through the expression of dn-SNARE domain in astrocytes, leads to the loss of BDNF effect upon LTP under mild stimulation conditions. These results suggest that astrocytes are able to respond differently to diverse intensities of stimulation, and in this way being able to dynamically control BDNF effect on synaptic plasticity.

\section{Discussion}


The main finding of the present study is that astrocytes play an active role in the facilitatory action of BDNF upon hippocampal LTP. In the three studied animal models, pharmacological astrocytic metabolism reduction in Wistar rats, IP3R2-/- mice (which lacks $\mathrm{Ca}^{2+}$ signaling in astrocyte) and dn-SNARE transgenic mice (which have astrocytic gliotransmitters release compromised), the effect of BDNF upon LTP was completely abolished, while inducing LTP with a mild- $\theta$-burst stimulation paradigm.

Astrocytes are critical players in diverse aspects of brain function and modulate several cognitive processes, such as learning and memory (Kofuji et al., 2021). Indeed, astrocytes by the $\mathrm{Ca}^{2+}$-dependent release of gliotransmitters modulate synaptic plasticity, the physiological basis of learning and memory(Kofuji et al., 2021; Pascual et al., 2005; Henneberger et al., 2010). In the present study we observed an impairment of hippocampal LTP while blocking astrocytes pharmacologically, for both strong and mild$\theta$-burst stimulation, as well as a decrease in the LTP magnitude in IP3R2-/- mice and dnSNARE (DOX-) mice, being our results in agreement with literature. Astrocytes modulate LTP, through the release and regulation of different gliotransmitters (Kofuji et al., 2021; Gonçalves-Ribeiro et al., 2019), namely glutamate, D-serine and ATP. Indeed, CA1 hippocampal LTP can be triggered when postsynaptic activity and astrocytic $\mathrm{Ca2+-}$ dependent glutamate release occur at the same time (Perea et al., 2007); this form of LTP is independent of postsynaptic NMDAR-mediated signaling and requires mGluR activation (Perea et al., 2007). The $\mathrm{Ca}^{2+}$-dependent release of $\mathrm{D}$-serine from CA1 hippocampal astrocytes controls NMDAR-dependent plasticity in the excitatory synapses nearby (Henneberger et al., 2010). The extracellular adenosine, derived from astrocytic ATP, regulates synaptic transmission and modulates LTP (Pascual et al., 2005). In spite of some controversy on the physiological relevance of $\mathrm{Ca}^{2+}$-dependent exocytosis of gliotransmitters, several studies reported a $\mathrm{Ca}^{2+}$-dependent exocytosis of glutamate and ATP from cultured astrocytes (Bal-Price et al., 2002; Petrelli et al., 2016). Recent evidence (Lalo et al., 2014) strongly reinforces the idea that ATP release from astrocytes has a relevant role in astrocytic-to-neuron signaling, namely: 1) the existence of a $\mathrm{Ca}^{2+}$ dependent mechanism leading to vesicular release of gliotransmitters from neocortical astrocytes, 2) the quantal activation of P2X receptors in neocortical neurons by ATP released from astrocytes, and 3 ) the glia-driven purinergic modulation of GABAergic transmission that is impaired by astrocytic expression of dn-SNARE (which precludes gliotransmitter release) or deletion of P2X4 receptors.

Brain-derived neurotrophic factor is a key neurotrophin which plays a critical modulatory action upon neuronal survival, dendritic growth and plasticity (Gottschalk et al., 1999; Carvalho et al., 2008; Minichiello, 2009; Graciano Leal et al., 2015). In the present work 
we also observed a potentiation of LTP magnitude in the presence of BDNF, nevertheless the effect was only observed when inducing LTP with a strong $\theta$-burst stimulation, and it was not observed while using a mild $\theta$-burst stimulation. Mainly, BDNF mediated is actions through the activation of TrkB-FL receptors, which are coupled to three different signaling pathways: (i) the phosphatidylinositol-3- kinase (PI3K)/Akt pathway, (ii) the Ras/MAPK pathway, and (iii) the PLCY pathway (Gottschalk et al., 1999). Working together, these pathways underlie important cognitive processes, including learning and memory formation. In fact, it is known that BDNF leads to an increase in LTP magnitude, by its binding to TrkB-FL receptors, both at a presynaptic and postsynaptic level (Figurov et al., 1996). Several studies reported that BNDF effect on LTP is depend of adenosine A2A receptor (Fontinha et al., 2008), being the excitatory action of BDNF on hippocampal LTP fully dependent on the recruitment and activation of A2AR, with the following activation of cAMP/protein kinase A (PKA) signaling cascade (Fontinha et al., 2008). Thus, adenosine released by astrocytes may account for the BDNF excitatory effect on LTP only for mild $\theta$-burst stimulation, while under strong $\theta$ burst stimulation the necessary adenosine for BDNF effect is provided by astrocytes. Thus we are showing that astrocytes can detect and decode differences in neuronal activity and respond to it by the release of gliotransmitters. Since BNDF action upon LTP is depend of $A 2 A$ receptors is possible that the gliotransmitters involved in this process is ATP/adenosine, and this remains to be evaluated. In this study, the use of different stimulation protocols allows to decipher the distinct astrocytic actions upon different stimulation contexts, where for mild- $\theta$-burst induced hippocampal LTP, astrocytic gliotransmitters would be found to be essential for the facilitatory BDNF action. However, for a strong- $\theta$-burst paradigm, astrocytic gliotransmitter were not necessary for BDNF action upon LTP. Therefore, in agreement with previous literature (Covelo et al., 2018), the present study presents evidences that astrocytes can detect and decode neuronal activity and respond distinctly to it.

Hence, this data shows for the first time that astrocytes play an active role in the facilitatory action of BDNF upon LTP and suggests that they do so by being a source of the gliotransmitters. 


\section{Acknowledgments}

This work was supported by project funding from Fundação para a Ciência e para a Tecnologia (FCT) to SHV (PTDC/BTM-SAL/32147/2017) and AMS (PTDC/MEDFAR/30933/2017). This project has received funding from H2020-WIDESPREAD-052017-Twinning (EpiEpinet) under grant agreement No. 952455. AAM (PD/BD/114278/2016), JG-R (PD/BD/150342/2019), and SP (SFRH/BD/147277/2019) are supported by $\mathrm{PhD}$ fellowships from FCT.

We thank Regeneron Pharmaceuticals for the gift of brain-derived neurotrophic factor.

The authors are grateful to Prof. Philip Haydon, Prof. Ju Chen and Prof João Oliveira for sharing the dn-SNARE and IP3R2 mice lines. 


\section{References}

Albensi, Benedict C., Oliver, Derek R., Toupin, Justin, and Odero, Gary. (2007).

Electrical Stimulation Protocols for Hippocampal Synaptic Plasticity and Neuronal Hyper-Excitability: Are They Effective or Relevant? Experimental Neurology, 204, $1-13$.

Araque, Alfonso, Parpura, Vladimir, Sanzgiri, Rita P., and Haydon, Philip G. (1999).

Tripartite Synapses: Glia, the Unacknowledged Partner. Trends in Neurosciences,.

Bal-Price, Anna, Moneer, Zahid, and Brown, Guy C. (2002). Nitric Oxide Induces Rapid, Calcium-Dependent Release of Vesicular Glutamate and ATP from Cultured Rat Astrocytes. Glia, 40, 312-23.

Berg-Johnsen, J., Paulsen, R. E., Fonnum, F., and Langmoen, I. A. (1993). Changes in

Evoked Potentials and Amino Acid Content during Fluorocitrate Action Studied in Rat Hippocampal Cortex. Experimental Brain Research, 96, 241-46.

Bliss, T. V.P., and Collingridge, G. L. (1993). A Synaptic Model of Memory: Long-Term Potentiation in the Hippocampus. Nature,.

Carvalho, a L, Caldeira, M V, Santos, S D, and Duarte, C B. (2008). Role of the BrainDerived Neurotrophic Factor at Glutamatergic Synapses. British Journal of Pharmacology, 153 Suppl, S310-24.

Covelo, Ana, and Araque, Alfonso. (2018). Neuronal Activity Determines Distinct

Gliotransmitter Release from a Single Astrocyte. eLife, 7, 1-19.

Figurov, Alexander, Pozzo-Miller, Lucas D., Olafsson, Petur, Wang, Ti, and Lu, Bai. (1996). Regulation of Synaptic Responses to High-Frequency Stimulation and LTP by Neurotrophins in the Hippocampus. Nature, 381, 706-9.

Fontinha, B M, Diógenes, M J, Ribeiro, J a, and Sebastião, a M. (2008). Enhancement of Long-Term Potentiation by Brain-Derived Neurotrophic Factor Requires Adenosine A2A Receptor Activation by Endogenous Adenosine.

Neuropharmacology, 54, 924-33.

Gonçalves-Ribeiro, Joana, Pina, Carolina Campos, Sebastião, Ana Maria, and Vaz, Sandra Henriques. (2019). Glutamate Transporters in Hippocampal LTD/LTP: Not Just Prevention of Excitotoxicity. Frontiers in Cellular Neuroscience,.

Gottschalk, W.A., Jiang, H., Tartaglia, N., Feng, L., Figurov, A., and Lu, B. (1999).

Signaling Mechanisms Mediating BDNF Modulation of Synaptic Plasticity in the 
Hippocampus. Learn.Mem., 6, 243-56.

Hassel, Bjørnar, Sonnewald, Ursula, Unsgård, Geirmund, and Fonnum, Frode. (1994). NMR Spectroscopy of Cultured Astrocytes: Effects of Glutamine and the Gliotoxin Fluorocitrate. Journal of Neurochemistry, 62, 2187-94.

Henneberger, Christian, Papouin, Thomas, Oliet, Stéphane H R, and Rusakov, Dmitri a. (2010). Long-Term Potentiation Depends on Release of D-Serine from Astrocytes. Nature, 463, 232-36.

Jerónimo-Santos, André, Vaz, Sandra Henriques, Parreira, Sara, Rapaz-Lérias, Sofia, Caetano, António P, Buée-Scherrer, Valérie, Castrén, Eero, et al. (2014). Dysregulation of TrkB Receptors and BDNF Function by Amyloid- $\beta$ Peptide Is Mediated by Calpain. Cerebral Cortex (New York, N.Y. : 1991),.

Kofuji, Paulo, and Araque, Alfonso. (2021). Astrocytes and Behavior. Annual Review of Neuroscience, $\mathbf{4 4}$, .

Korte, Martin, Carroll, Patrick, Wolf, Eckhard, Brem, Gottfried, Thoenen, Hans, and Bonhoeffer, Tobias. (1995). Hippocampal Long-Term Potentiation Is Impaired in Mice Lacking Brain-Derived Neurotrophic Factor. Proceedings of the National Academy of Sciences of the United States of America, 92, 8856-60.

Lalo, Ulyana, Palygin, Oleg, Rasooli-Nejad, Seyed, Andrew, Jemma, Haydon, Philip G., and Pankratov, Yuriy. (2014). Exocytosis of ATP From Astrocytes Modulates Phasic and Tonic Inhibition in the Neocortex. PLoS Biology, 12, .

Largo, Carlota, Cuevas, Pedro, Somjen, George G., Martín Del Río, Rafael, and Herreras, Oscar. (1996). The Effect of Depressing Glial Function in Rat Brain in Situ on Ion Homeostasis, Synaptic Transmission, and Neuron Survival. Journal of Neuroscience, 16, 1219-29.

Leal, G., Bramham, C. R., and Duarte, C. B. (2017). BDNF and Hippocampal Synaptic Plasticity. In Vitamins and Hormones, 104:153-95.

Leal, Graciano, Afonso, Pedro M., Salazar, Ivan L., and Duarte, Carlos B. (2015).

Regulation of Hippocampal Synaptic Plasticity by BDNF. Brain Research, 1621, 82-101.

Li, Xiaodong, Zima, Aleksey V., Sheikh, Farah, Blatter, Lothar a., and Chen, Ju. (2005). Endothelin-1-Induced Arrhythmogenic Ca2+ Signaling Is Abolished in Atrial Myocytes of Inositol-1,4,5-trisphosphate(IP3)-Receptor Type 2-Deficient Mice. Circulation Research, 96, 1274-81.

Minichiello, Liliana. (2009). TrkB Signalling Pathways in LTP and Learning. Nature 
Reviews. Neuroscience, 10, 850-60.

Minichiello, Liliana, Calella, Anna Maria, Medina, Diego L., Bonhoeffer, Tobias, Klein, Rüdiger, and Korte, Martin. (2002). Mechanism of TrkB-Mediated Hippocampal Long-Term Potentiation. Neuron, 36, 121-37.

Pascual, Olivier, Casper, Kristen B, Kubera, Cathryn, Zhang, Jing, Revilla-Sanchez, Raquel, Sul, Jai-Yoon, Takano, Hajime, Moss, Stephen J, McCarthy, Ken, and Haydon, Philip G. (2005). Astrocytic Purinergic Signaling Coordinates Synaptic Networks. Science (New York, N.Y.), 310, 113-16.

Patterson, Susan L., Abel, Ted, Deuel, Thomas A.S., Martin, Kelsey C., Rose, Jack C., and Kandel, Eric R. (1996). Recombinant BDNF Rescues Deficits in Basal Synaptic Transmission and Hippocampal LTP in BDNF Knockout Mice. Neuron, 16, 1137-45.

Paulsen, R. E., Contestabile, A., Villani, L., and Fonnum, F. (1987). An In Vivo Model for Studying Function of Brain Tissue Temporarily Devoid of Glial Cell Metabolism: The Use of Fluorocitrate. Journal of Neurochemistry, 48, 1377-85.

Perea, Gertrudis, and Araque, Alfonso. (2007). Astrocytes Potentiate Transmitter Release at Single Hippocampal Synapses. Science (New York, N.Y.), 317, 108386.

Petrelli, Francesco, and Bezzi, Paola. (2016). Novel Insights into Gliotransmitters. Current Opinion in Pharmacology,

Pins, Benoit De, Cifuentes-Díaz, Carmen, Thamila Farah, Amel, López-Molina, Laura, Montalban, Enrica, Sancho-Balsells, Anna, López, Ana, et al. (2019). Conditional BDNF Delivery from Astrocytes Rescues Memory Deficits, Spine Density, and Synaptic Properties in the 5xFAD Mouse Model of Alzheimer Disease. Journal of Neuroscience, 39, 2441-58.

Rei, N., Rombo, D. M., Ferreira, M. F., Baqi, Y., Müller, C. E., Ribeiro, J. A., Sebastião, A. M., and Vaz, S. H. (2020). Hippocampal Synaptic Dysfunction in the SOD1G93A Mouse Model of Amyotrophic Lateral Sclerosis: Reversal by Adenosine A2AR Blockade. Neuropharmacology, 171, .

Rose, Christine R, Blum, Robert, Pichler, Bruno, Lepier, Alexandra, Kafitz, Karl W, and Konnerth, Arthur. (2003). Truncated TrkB-T1 Mediates Neurotrophin-Evoked Calcium Signalling in Glia Cells. Nature, 426, 74-78.

Sardinha, Vanessa Morais, Guerra-Gomes, Sónia, Caetano, Inês, Tavares, Gabriela, Martins, Manuella, Reis, Joana Santos, Correia, Joana Sofia, et al. (2017). 
Astrocytic Signaling Supports Hippocampal-prefrontal Theta Synchronization and Cognitive Function. GLIA, 65, 1944-60.

Sebastião, Ana M, Assaife-Lopes, Natália, Diógenes, Maria J, Vaz, Sandra H, and Ribeiro, Joaquim a. (2011). Modulation of Brain-Derived Neurotrophic Factor (BDNF) Actions in the Nervous System by Adenosine A(2A) Receptors and the Role of Lipid Rafts. Biochimica et Biophysica Acta, 1808, 1340-49.

Sultan, Sébastien, Li, Liyi, Moss, Jonathan, Petrelli, Francesco, Cassé, Frédéric, Gebara, Elias, Lopatar, Jan, et al. (2015). Synaptic Integration of Adult-Born Hippocampal Neurons Is Locally Controlled by Astrocytes. Neuron, 88, 957-72.

Swanson, Raymond A., and Graham, Steven H. (1994). Fluorocitrate and Fluoroacetate Effects on Astrocyte Metabolism in Vitro. Brain Research, 664, 94100.

Todd, Keith J., Auld, Daniel S., and Robitaille, Richard. (2007). Neurotrophins Modulate Neuron-Glia Interactions at a Vertebrate Synapse. European Journal of Neuroscience, 25, 1287-96.

Xu, Baoji, Gottschalk, Wolfram, Chow, Ana, Wilson, Rachel I., Schnell, Eric, Zang, Keling, Wang, Denan, Nicoll, Roger A., Lu, Bai, and Reichardt, Louis F. (2000). The Role of Brain-Derived Neurotrophic Factor Receptors in the Mature Hippocampus: Modulation of Long-Term Potentiation through a Presynaptic Mechanism Involving trkB. Journal of Neuroscience, 20, 6888-97. 
Figure 1. BDNF effect upon hippocampal LPT is not under astrocytic control while inducing LTP with a strong- $\theta$-burst stimulation. In $(A)$ is represented the average time course changes in the fEPSP slope induced by strong $\theta$-burst stimulation in Wistar rats in the absence $(\bullet)(n=5)$ or in the presence $(\bullet)$ of BDNF $(20 \mathrm{ng} / \mathrm{mL})(n=5)$. In $(B)$ is represented the average time course changes in the fEPSP slopes induced by strong $\theta-$ burst stimulation in Wistar rats in the presence of $F C$ alone $(\bullet)(n=5)$ or together with BDNF $(\bullet)(20 \mathrm{ng} / \mathrm{mL})(\mathrm{n}=6)$. In $(\mathrm{C})$ is represented the histogram depict LTP magnitude (change in fEPSP slope at 50-60 min) induced by strong $\theta$-burst stimulation in Wistar rats in the presence or absence of FC and/or BDNF as indicated below each column. (D) represents the time course of averaged normalized changes in fEPSP slope after delivery (arrow) of a strong $\theta$-burst to hippocampal slices from IP3R2 ${ }^{++}$in the absence $(\bullet)(n=8)$ or in the presence $(\bullet)$ of BDNF $(20 \mathrm{ng} / \mathrm{mL})(\mathrm{n}=7)$. (E) Represents the time course of averaged normalized changes in fEPSP slope after delivery (arrow) of a strong $\theta$-burst to hippocampal slices from IP3R2 ${ }^{-/-}$in the absence $(\bullet)(n=4)$ or in the presence (•) of BDNF $(20 \mathrm{ng} / \mathrm{mL})(\mathrm{n}=3)$. (F) corresponds to the histogram depict LTP magnitude (change in fEPSP slope at 50-60 min) induced by strong $\theta$-burst stimulation in the absence or presence of BDNF (20 ng/mL) for each group of animals tested, as indicated below each column. (G) Represents the time course of averaged normalized changes in fEPSP slope after delivery (arrow) of a strong $\theta$-burst to hippocampal slices from WT $($ Dox +$)$ in the absence $(\bullet)(n=8)$ or in the presence $(\bullet)$ of BDNF $(20 \mathrm{ng} / \mathrm{mL})(n=5) .(H)$ Represents the time course of averaged normalized changes in fEPSP slope after delivery (arrow) of a strong $\theta$-burst to hippocampal slices from dn-SNARE (Dox -) in the absence $(\bullet)(n=6)$ or in the presence $(\bullet)$ of BDNF $(20 \mathrm{ng} / \mathrm{mL})(n=4)$. (I) corresponds to the histogram depict LTP magnitude (change in fEPSP slope at 50-60 min) induced by strong $\theta$-burst stimulation in the absence or presence of BDNF $(20 \mathrm{ng} / \mathrm{mL})$ for each group of animals tested, as indicated below each column. The ordinates in (A), (B), (D), (E), $(\mathrm{G})$ and $(\mathrm{H})$ represent normalized fEPSP slopes where $0 \%$ corresponds to the averaged slope recorded for 10 min before $\theta$-burst stimulation and the abscissa represents the time that average begun. Insets bellow each graph correspond to illustrative traces from representative experiments; each trace is the average of eight consecutive responses obtained for each group before ( $\cdots)$ and 58-60 min after (-) $\theta$-burst stimulation, and is composed of the stimulus artifact, followed by the presynaptic volley and the fEPSP. All values are presented as mean \pm standard error of mean (SEM) from $n$ independent observations. n.s (not significant): $p>0.05 ;{ }^{*} p \leq 0.05 ;{ }^{* *} p \leq 0.01 ;{ }^{\theta} p \leq 0.01$; ${ }^{\# \#} p \leq 0.001$ (one-way ANOVA followed by Holm-Sidak's post hoc test for multiple comparisons). 
Figure 2. Astrocytic signaling mediates BDNF action under mild- $\theta$-burst induced LTP. In (A) is represented the average time course changes in the fEPSP slope induced by mild $\theta$-burst stimulation in Wistar rats in the absence $(\bullet)(n=7)$ or in the presence $(\bullet)$ of BDNF $(20 \mathrm{ng} / \mathrm{mL})(\mathrm{n}=6)$. In $(B)$ is represented the average time course changes in the fEPSP slopes induced by mild $\theta$-burst stimulation in Wistar rats in the presence of FC alone $(\bullet)(n=12)$ or together with BDNF $(\bullet)(20 \mathrm{ng} / \mathrm{mL})(\mathrm{n}=7)$. In (C) is represented the histogram depict LTP magnitude (change in fEPSP slope at 50-60 min) induced by mild $\theta$-burst stimulation in Wistar rats in the presence or absence of FC and/or BDNF as indicated below each column. (D) represents the time course of averaged normalized changes in fEPSP slope after delivery (arrow) of a mild $\theta$-burst to hippocampal slices from IP3R2 ${ }^{+/+}$in the absence $(\bullet)(n=7)$ or in the presence $(\bullet)$ of BDNF $(20 \mathrm{ng} / \mathrm{mL})(\mathrm{n}=$ 7). (E) Represents the time course of averaged normalized changes in fEPSP slope after delivery (arrow) of a mild $\theta$-burst to hippocampal slices from IP3R2 ${ }^{-/}$in the absence $(\bullet)$ $(n=7)$ or in the presence $(\bullet)$ of BDNF $(20 \mathrm{ng} / \mathrm{mL})(n=7)$. (F) corresponds to the histogram depict LTP magnitude (change in fEPSP slope at 50-60 min) induced by mild $\theta$-burst stimulation in the absence or presence of BDNF $(20 \mathrm{ng} / \mathrm{mL})$ for each group of animals tested, as indicated below each column. (G) Represents the time course of averaged normalized changes in fEPSP slope after delivery (arrow) of a mild $\theta$-burst to hippocampal slices from WT (Dox +$)$ in the absence $(\bullet)(n=4)$ or in the presence $(\bullet)$ of BDNF (20 ng/mL) $(n=4)$. (H) Represents the time course of averaged normalized changes in fEPSP slope after delivery (arrow) of a mild $\theta$-burst to hippocampal slices from dn-SNARE (Dox -$)$ in the absence $(\bullet)(n=4)$ or in the presence $(\bullet)$ of BDNF $(20$ $\mathrm{ng} / \mathrm{mL})(\mathrm{n}=3)$. (I) corresponds to the histogram depict LTP magnitude (change in fEPSP slope at 50-60 $\mathrm{min}$ ) induced by mild $\theta$-burst stimulation in the absence or presence of BDNF (20 ng/mL) for each group of animals tested, as indicated below each column. The ordinates in $(A),(B),(D),(E),(G)$ and $(H)$ represent normalized fEPSP slopes where $0 \%$ corresponds to the averaged slope recorded for $10 \mathrm{~min}$ before $\theta$-burst stimulation and the abscissa represents the time that average begun. Insets bellow each graph correspond to illustrative traces from representative experiments; each trace is the average of eight consecutive responses obtained for each group before ( $\cdots$ ) and 58-60 min after (-) $\theta$-burst stimulation, and is composed of the stimulus artifact, followed by the presynaptic volley and the fEPSP. All values are presented as mean \pm standard error of mean (SEM) from $n$ independent observations. ${ }^{*} p \leq 0.05 ;{ }^{* *} p \leq 0.01 ;{ }^{\theta} p \leq 0.01 ;{ }^{\# \#} p \leq 0.01$ (one-way ANOVA followed by Holm-Sidak's post hoc test for multiple comparisons). 
Supplementary Figure 1. BDNF effect over LTP is dependent on the astrocyticrelease of gliotransmitters via exocytosis. (A) Represents the time course of averaged normalized changes in fEPSP slope after delivery (arrow) of a strong $\theta$-burst to hippocampal slices from dn-SNARE $(\operatorname{Dox}+)$ in the absence $(\bullet)(n=5)$ or in the presence $(\bullet)$ of BDNF $(20 \mathrm{ng} / \mathrm{mL})(n=3)$. (B) corresponds to illustrative traces from representative experiments; each trace is the average of eight consecutive responses obtained for each group before ( $\cdots)$ and 58-60 min after $(-) \theta$-burst stimulation, and is composed of the stimulus artifact, followed by the presynaptic volley and the fEPSP. (C) corresponds to the histogram depict LTP magnitude (change in fEPSP slope at 50-60 $\mathrm{min}$ ) induced by strong $\theta$-burst stimulation in hippocampal slices from dn-SNARE (Dox $+)$ mice in the presence or absence of BDNF $(20 \mathrm{ng} / \mathrm{mL})$, as indicated below each column. (D) Represents the time course of averaged normalized changes in fEPSP slope after delivery (arrow) of a mild $\theta$-burst to hippocampal slices from dn-SNARE (Dox + ) in the absence $(\bullet)(n=4)$ or in the presence $(\bullet)$ of BDNF $(20 \mathrm{ng} / \mathrm{mL})(n=4)$. (B) correspond to illustrative traces from representative experiments; each trace is the average of eight consecutive responses obtained for each group before ( $\cdots)$ and 58-60 min after (-) $\theta$-burst stimulation, and is composed of the stimulus artifact, followed by the presynaptic volley and the fEPSP. (C) corresponds to the histogram depict LTP magnitude (change in fEPSP slope at 50-60 min) induced by mild $\theta$-burst stimulation in hippocampal slices from dn-SNARE (Dox + ) mice in the presence or absence of BDNF $(20 \mathrm{ng} / \mathrm{mL})$, as indicated below each column. The ordinates in (A) and (D) represent normalized fEPSP slopes where $0 \%$ corresponds to the averaged slope recorded for $10 \mathrm{~min}$ before $\theta$-burst stimulation and the abscissa represents the time that average begun. Insets bellow each graph correspond to illustrative traces from representative experiments; each trace is the average of eight consecutive responses obtained for each group before ( $\cdots)$ and 58-60 min after (-) $\theta$-burst stimulation, and is composed of the stimulus artifact, followed by the presynaptic volley and the fEPSP. All values are presented as mean \pm standard error of mean (SEM) from $n$ independent observations. Statistical significance was assessed by unpaired t-test between control $(\bullet)$ and $\operatorname{BDNF}(20 \mathrm{ng} / \mathrm{mL})(\bullet)$ conditions. $\mathrm{p}>0.05$; ${ }^{*} \mathrm{p} \leq$ 0.05 . 
A
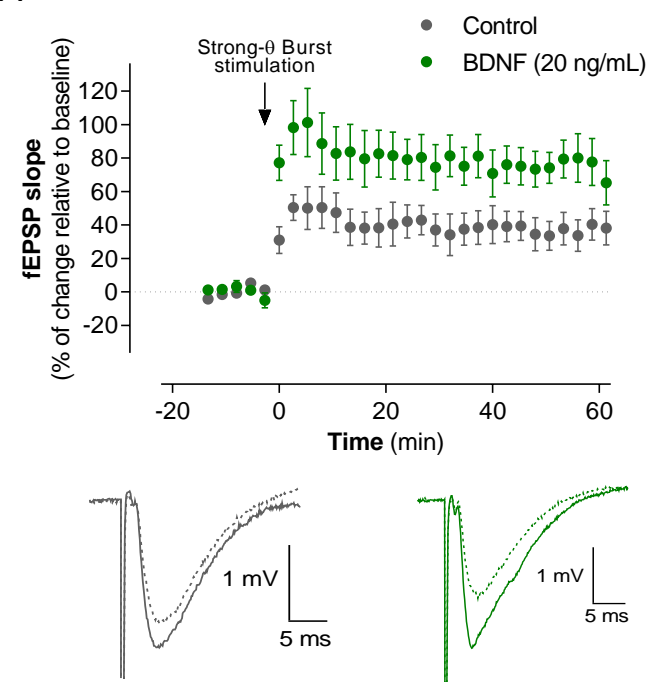

.... Before LTP

- After LTP

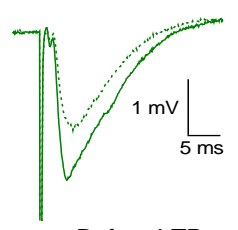

... Before LTP

- After LTP
B

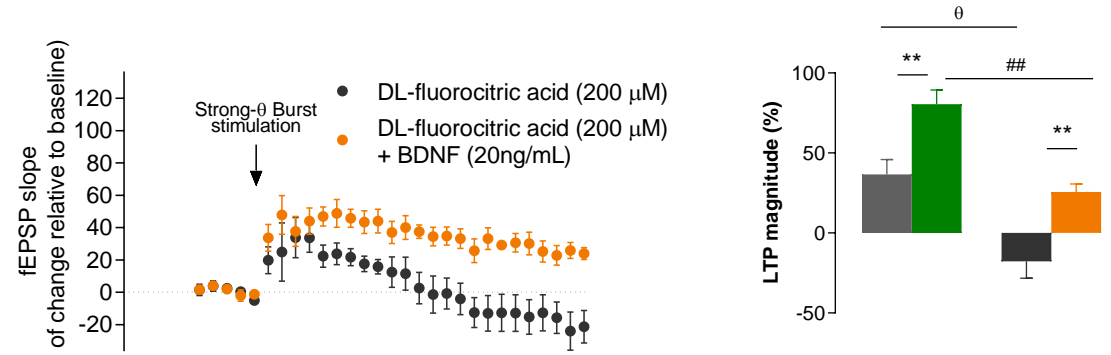

C

BDNF $(20 \mathrm{ng} / \mathrm{mL})$

DL-fluorocitric acid (200

$\mu \mathrm{M})$
D

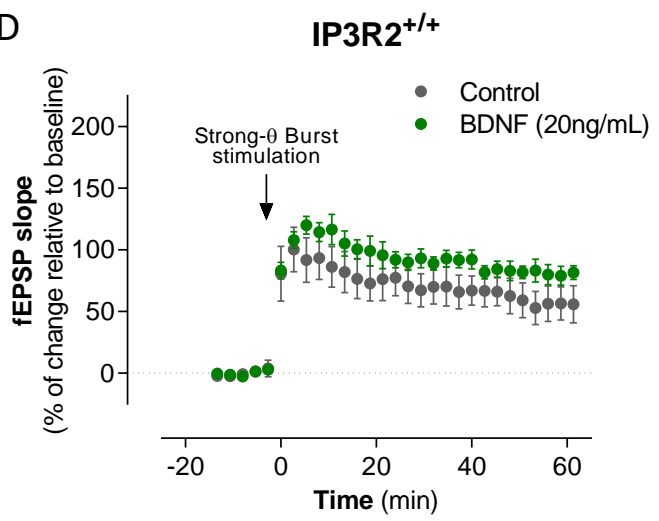

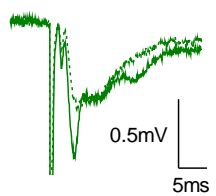

.... Before LTP

— After LTP

... Before LTP

- After LTP

E
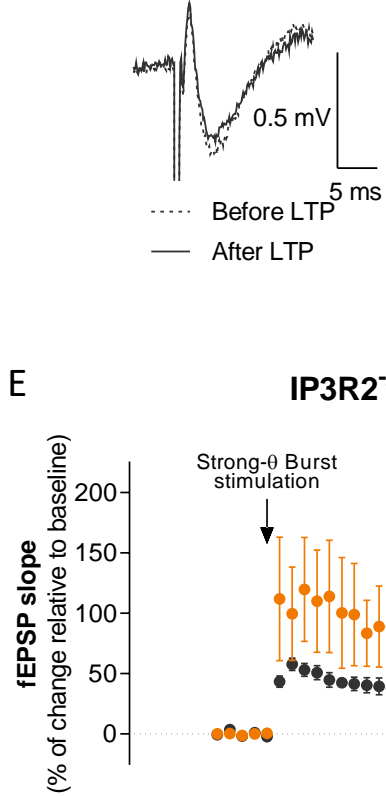

— After LTP
IP3R2 $2^{-/-}$

- Control

- $\operatorname{BDNF}(20 \mathrm{ng} / \mathrm{mL})$
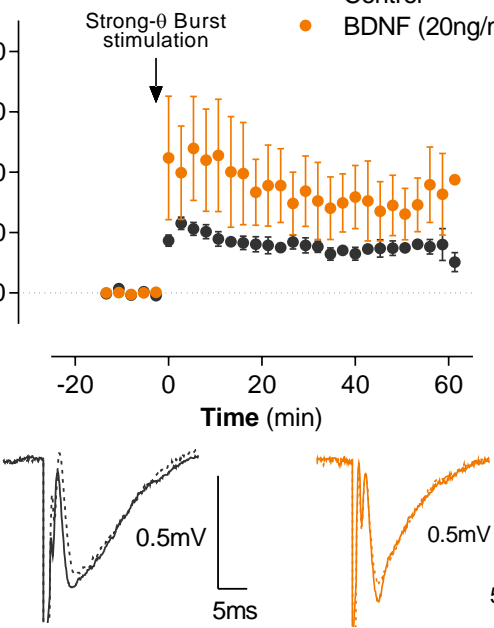

.... Before LTP

- After LTP

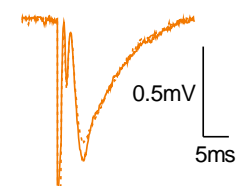

Before LTP

- After LTP
G
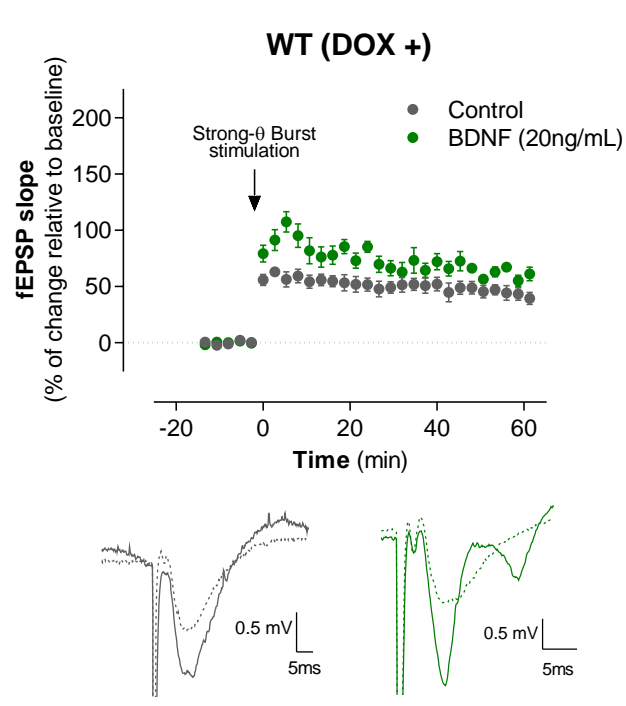

B.. Before LTP

— After LTP

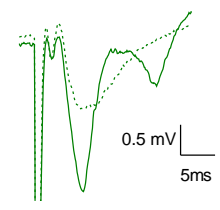

... Before LTP
$\mathrm{H}$

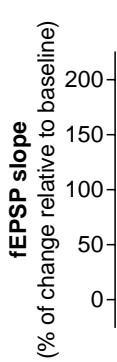

dn-SNARE (DOX -)
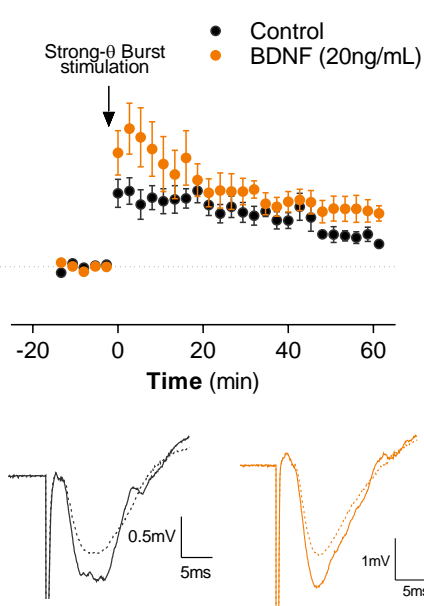

… Before LTP

- After LTP
F

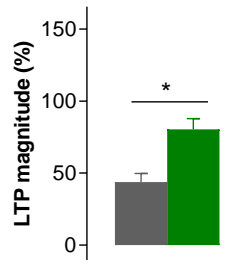

BDNF $(20 \mathrm{ng} / \mathrm{ml})$

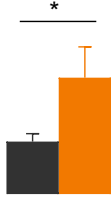

$\frac{-}{\text { IP3R2 }}$

\section{Figure 1}


A
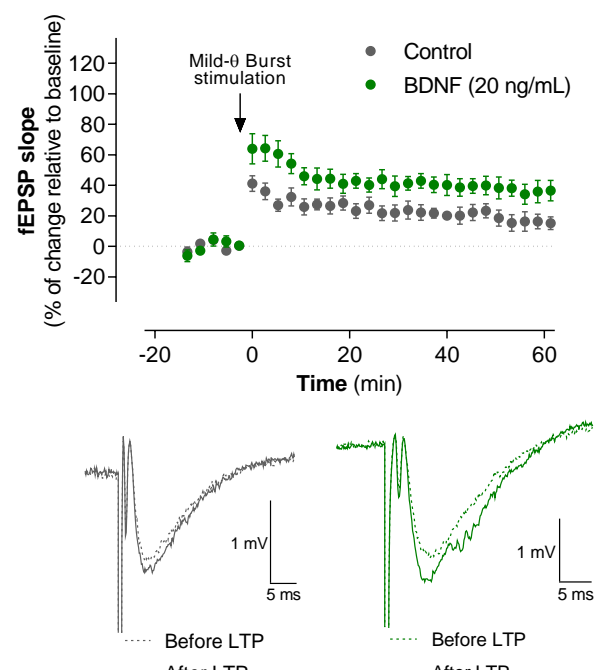

- After LTP

- After LTP

D

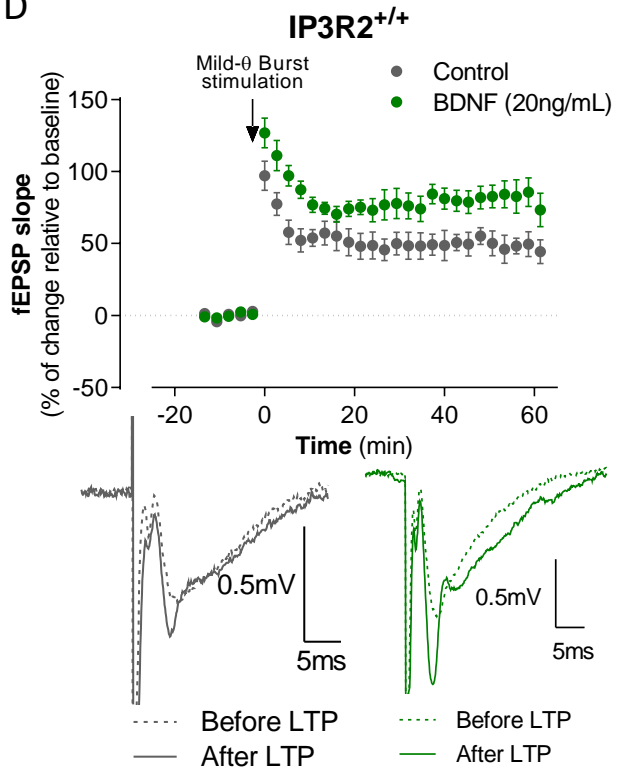

G
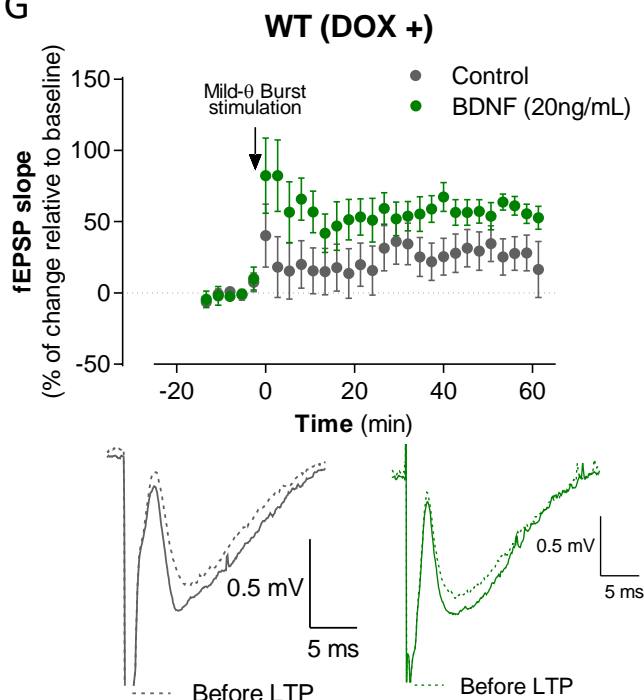

— After LTP

— After LTP
B
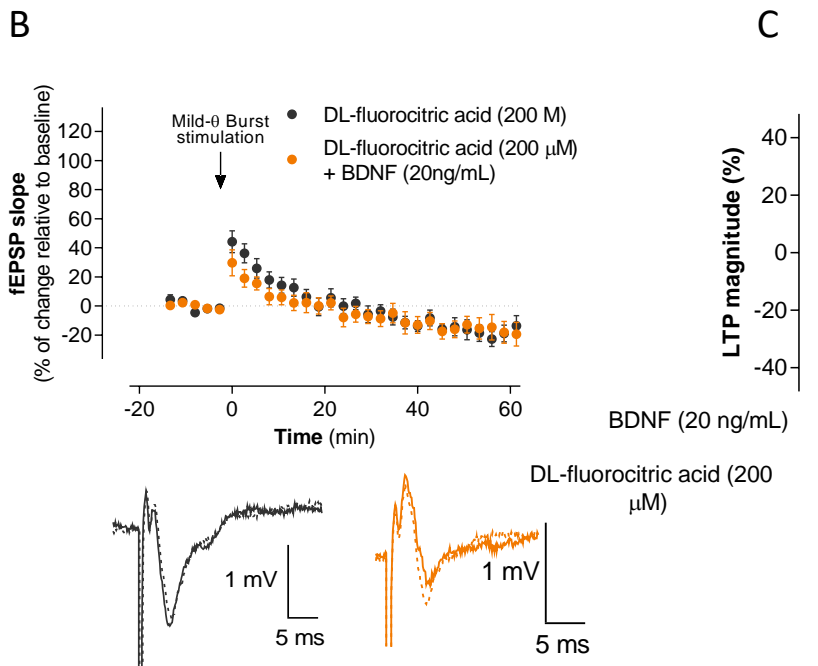

Before LTP

Before LTP

- After LTP

— After LTP

E

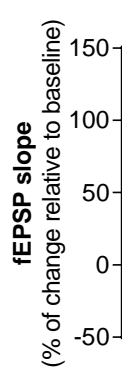

IP3R2-/-

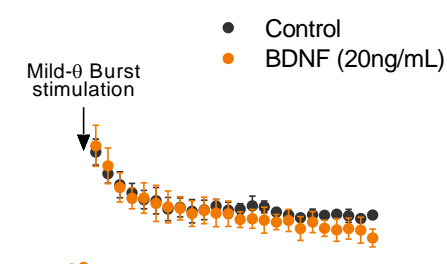

F

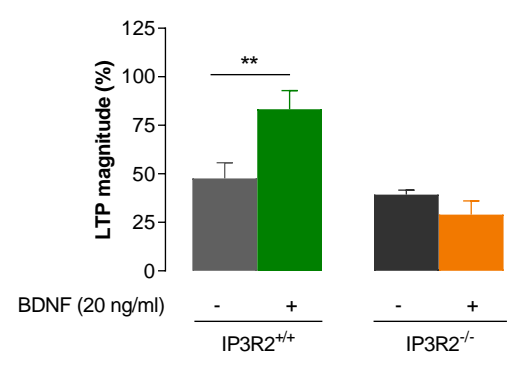

$\mu \mathrm{M})$

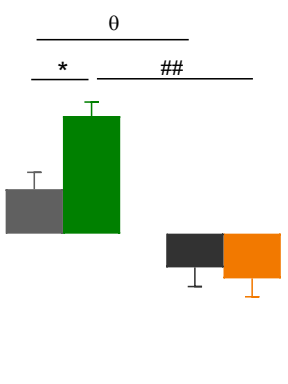

BDNF $(20 \mathrm{ng} / \mathrm{mL})$

$$
\text { (200 }
$$

- +

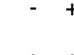

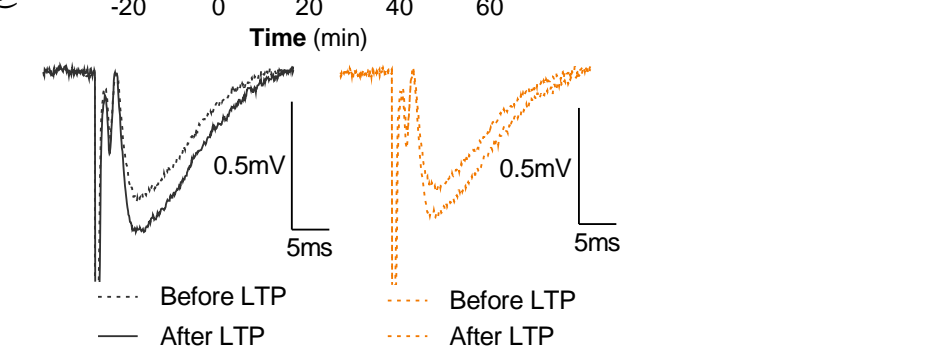
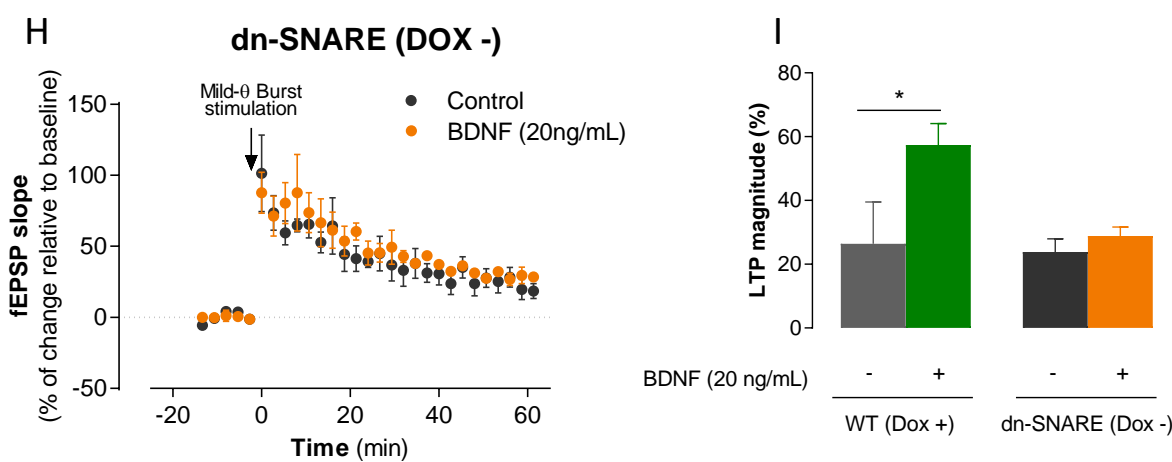

Figure 2

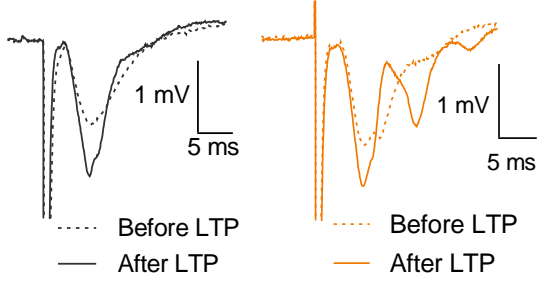

BDNF $(20 \mathrm{ng} / \mathrm{mL})$

WT (Dox +) 
A

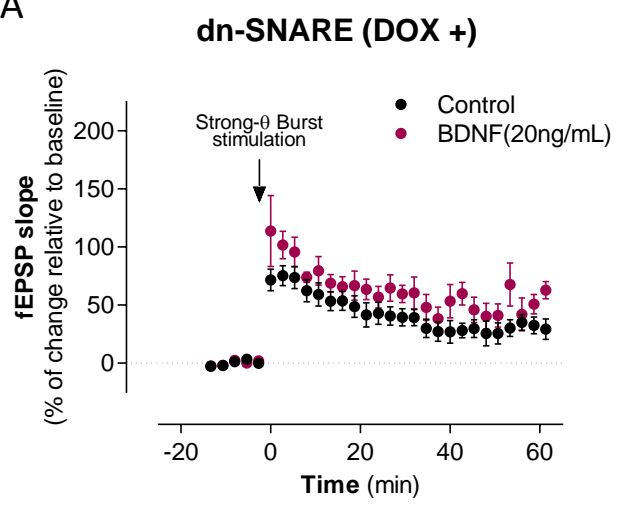

D

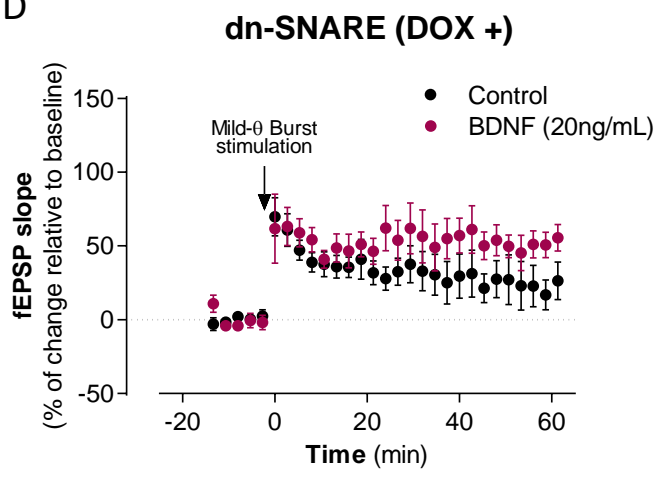

B

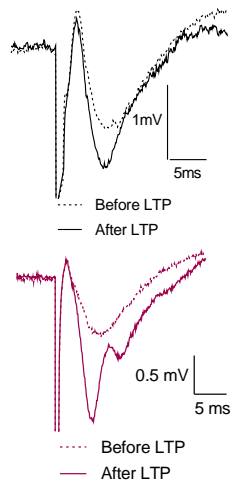

E

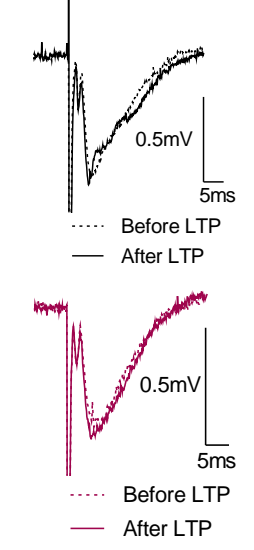

C

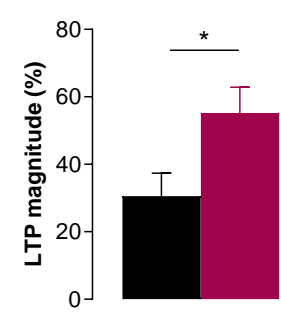

BDNF $(20 \mathrm{ng} / \mathrm{mL})$ dn-SNARE (Dox +)

F

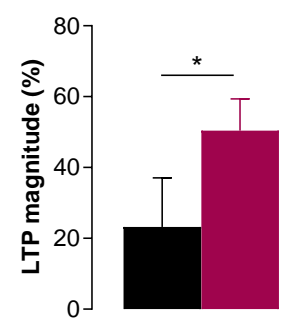

BDNF $(20 \mathrm{ng} / \mathrm{mL})$ dn-SNARE (Dox + ) 